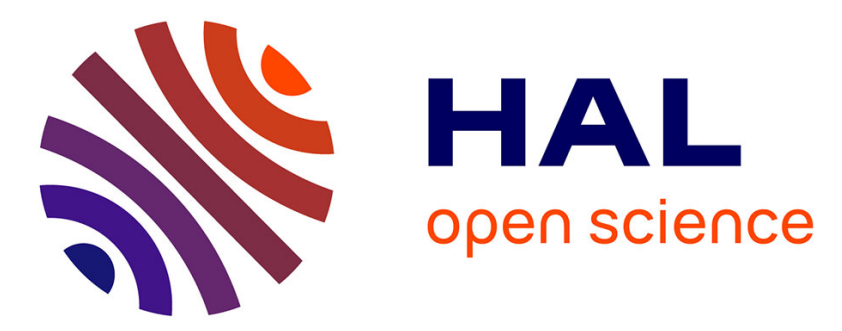

\title{
A Mixed Strategy for a Competitive Game in Delay Tolerant Networks
}

\author{
Thi Thu Hang Nguyen, Olivier Brun, Balakrishna Prabhu
}

\section{To cite this version:}

Thi Thu Hang Nguyen, Olivier Brun, Balakrishna Prabhu. A Mixed Strategy for a Competitive Game in Delay Tolerant Networks. Computer Performance Engineering - 15th European Workshop, EPEW 2018, Paris, France, October 29-30, 2018, Proceedings, pp.190-204, 2018, 10.1007/978-3-030-02227-

3_13. hal-02062162

\author{
HAL Id: hal-02062162 \\ https://hal.laas.fr/hal-02062162
}

Submitted on 8 Mar 2019

HAL is a multi-disciplinary open access archive for the deposit and dissemination of scientific research documents, whether they are published or not. The documents may come from teaching and research institutions in France or abroad, or from public or private research centers.
L'archive ouverte pluridisciplinaire HAL, est destinée au dépôt et à la diffusion de documents scientifiques de niveau recherche, publiés ou non, émanant des établissements d'enseignement et de recherche français ou étrangers, des laboratoires publics ou privés. 


\title{
A mixed strategy for a competitive game in Delay Tolerant Networks
}

\author{
Thi Thu Hang Nguyen ${ }^{1,2}$, Olivier Brun ${ }^{1}$, and Balakrishna Prabhu ${ }^{1}$ \\ 1 LAAS-CNRS, Université de Toulouse, CNRS, Toulouse, France \\ ${ }^{2}$ Université de Toulouse, INSA, Toulouse, France \\ \{thnguye, brun, bala\}@laas.fr
}

\begin{abstract}
We consider a non-cooperative game between $N$ relays in Delay Tolerant Networks with one fixed source and one fixed destination. The source has no contact with the destination, so it has to rely on the relays when it has a message to send. We assume that the source has a sequence of messages and it proposes them to relays one by one with a fixed reward for the first transmission for each message. We analyse a symmetric mixed strategy for this game. A mixed strategy means a relay decides to accept relaying the $k^{t h}$ message with probability $q_{k}$ when it meets the source. We establish the conditions under which $q_{k}=1$; $q_{k}=0$ or $q_{k} \in(0,1)$, and prove the existence and the uniqueness of the symmetric Nash equilibrium. We also give the formula to compute this mixed strategy as well as the probability of success and the delay of a given message. When $k$ is large, we give the limiting value of the mixed strategy $q$ and the probability of success for the messages.
\end{abstract}

Keywords: Delay Tolerant Networks · Incentive mechanism · Stochastic game.

\section{Introduction}

In Delay Tolerant Networks (DTN) [3,9-11], the approach used by mobile nodes to communicate in the absence of a communication infrastructure is based on the so-called store-carry-forward paradigm: a source node gives a copy of its message to all mobile nodes that it meets, asking them to keep it until they can forward it to the destination. Although other routing schemes have been proposed $[13,15]$, in this work we shall specifically consider two-hop routing DTNs $[2,21]$, in which once a relay has the message, it can only transmit it to the destination.

The above approach implicitly assumes that mobile nodes accept to use their scarce energy resources for relaying messages of others out of altruism. In practice, it can be expected that some nodes will act as free-riders, that is, that they will use the network to send their own messages without offering their resources in exchange for relaying the messages of others. Clearly, if there are too many selfish nodes in a DTN, the network collapses and mobile nodes can no longer communicate with one another. A central issue in DTNs is therefore to convince mobile nodes to relay messages. Many incentive mechanisms have 
been proposed to avoid the free-rider problem in DTNs, including reputationbased schemes $[12,16,22-24]$, barter-based schemes $[4,5,20]$ and credit-based schemes $[7,8,14,19,25,26]$. In contrast to most of the incentive mechanisms proposed in the literature, explicit guarantees on the probability of message delivery and on the mean time to deliver a message have been obtained for the credit-based scheme considered in $[17,18]$ (see also [19] for a closely-related mechanism).

The authors of $[17,18]$ consider a source which promises a fixed reward to the relay who first delivers a message to the destination. The source is backlogged and only one message at a time is proposed by the source. Inter-contact times of relays with the source and the destination are exponentially distributed. When it meets the source, a relay has the choice to either accept the message or not, and if it accepts, it can decide to drop the message at any time in the future at no additional cost. The competition between relays is modelled as a stochastic game in which each relay seeks to minimize its expected net cost, that is, the sum of its expected energy and storage costs minus its expected reward. It is proven that the optimal policy of a relay is of threshold type: it accepts a message until a first threshold $\theta$ and then keeps it until it either meets the destination or reaches a second threshold $\gamma$ (which can be infinite). Explicit formulas for computing the thresholds as well as the probability of message delivery are derived for the unique symmetric Nash equilibrium, in which all relays use the same thresholds and no player can benefit by unilaterally changing its policy.

The analysis in $[17,18]$ implicitly assumes that the source tells the relays when a message was proposed for the first time, or, in other words, when this message was generated. Our objective in the present paper is to understand whether it is profitable for the source to give this information to the relays. We thus consider the same incentive mechanism, but assuming that when it meets the source, a relay has to make its decision without knowing for how long the message is in circulation. The only information available to the relay is the value $R$ of the reward and the period of time $T$ during which the message is proposed by the source.

Since it does not know for how long a message is available, we assume that a relay decides to accept a message according to a randomized policy, that is, when relay $i$ meets the source, it accepts the $k^{t h}$ message with probability $q_{k}^{i}$, and rejects it with probability $1-q_{k}^{i}$. If it accepts the message, the relay keeps it until it reaches the destination. The value of $q_{k}^{i}$ is computed by relay $i$ so as to minimize its expected net cost, and it of course depends on $R$ and $T$, but also on the number of relays competing for the delivery of the $k^{\text {th }}$ message (some relays may be busy delivering previous messages). We note that a similar setting was considered in [1], but with a different cost structure and assuming that the source has only one message to transmit.

We establish under which condition $q_{k}^{i}>0$ for all $i$, and show that, under this condition, there exists a unique value $q_{k}>0$ such that if all relays accept the $k^{\text {th }}$ message with probability $q_{k}$, no relay has anything to gain by unilaterally changing its acceptance probability. In other words, the situation in which all 
relays accept the $k^{t h}$ message with probability $q_{k}$ corresponds to a symmetric Nash equilibrium, and this equilibrium is unique. Explicit expressions for the probability of message delivery and the mean time to deliver a message at the symmetric Nash equilibrium are then derived. Assuming that $q_{k}$ converges as $k \rightarrow \infty$, we also obtain an explicit characterization of the asymptotic value of the acceptance probability $q_{\infty}$. Finally, we compare the performance obtained with the threshold-type strategy in the full information setting and with the randomized policy in the no information setting.

The rest of this paper is organized as follows. Section 2 is devoted to model description. In Section 3, we establish the conditions for the existence and uniqueness of symmetric Nash equilibria and present a method for recursively computing the acceptance probabilities $q_{k}$. The asymptotic value of the acceptance probability is also derived in Section 3. Explicit expressions for the main performance metrics at the symmetric Nash equilibrium are then derived in Section 4. Finally, numerical results pertaining to the comparison of the full information setting and the no information setting are given in Section 5.

\section{Assumption and Model Description}

We consider a two-hop network of $N$ mobile nodes with one fixed source and one fixed destination. The source is backlogged, that is, it has an unlimited number of messages to send to the destination. Since the source and the destination are not in radio range of each other, the source cannot transmit its messages directly to the destination. Instead, it proposes a new message to the relays every $T$ units of time, promising a fixed reward $R$ to the first one to deliver the current message to the destination. We assume that the relays are moving randomly and that the inter-contact times of a given relay with the source (resp. destination) are i.i.d. and follow an exponential distribution with rate $\lambda$ (resp. $\mu$ ). This assumption holds (at least approximately) under the Random Waypoint Mobility model [6].

When it accepts a message, a relay incurs a one-time reception cost $C_{r}$ for receiving it from the source. There is then a cost $C_{s}$ per unit of time for keeping the message in its buffer. Finally, the relay incurs a transmission $\operatorname{cost} C_{d}$ for sending the message to the destination. We however assume that the latter cost is incurred by the relay if and only if the message has not been already delivered to the destination by another relay. If on the contrary the relay is the first one to deliver the message to the destination, it incurs the cost $C_{d}$ but gets the reward $R$. In the following, we define $\bar{R}=R-C_{d}$.

When it proposes the current message (say message $k$ ) to relay $i$, the source informs it of the values of $R$ and $T$, but does not tell it for how long the current message is available. The relay accepts message $k$ with probability $q_{k}^{i}$, and rejects it with probability $1-q_{k}^{i}$. If the $k^{t h}$ message was rejected by relay $i$, then this relay cannot accept it later on when it meets again the source. We also assume that if the relay accepts the message, it has to keep it until it meets the destination. Finally, we assume that a relay can store only one message at a time and cannot drop a message to accept a new one. 
Relay $i$ computes its acceptance probability $q_{k}^{i}$ so as to minimize its expected net cost, which depends on its probability to be the first one to deliver message $k$. Obviously, the latter probability in turn depends on the acceptance probabilities of the other relays. We say that a vector $\left(q_{k}^{1}, q_{k}^{2}, \ldots, q_{k}^{N}\right)$ is a Nash equilibrium if no relay $i$ can decrease its expected net cost by unilaterally changing its acceptance probability $q_{k}^{i}$. A symmetric Nash equilibrium is a Nash equilibrium for which $q_{k}^{i}=q_{k}$ for all $i$, for some value $q_{k}$. In the following, we shall specifically focus on symmetric Nash equilibria.

\section{Acceptance Probabilities under the Symmetric Nash Equilibrium}

\subsection{Acceptance Probabilities}

Consider a tagged relay and let us analyse the competition for the delivery of the $k^{t h}$ message. Assume that all other relays accept the $k^{t h}$ message with probability $q_{k}$. If the tagged relay accepts the message with probability $q_{k}^{\prime}$, its net expected cost is

$$
q_{k}^{\prime}\left(C_{r}+\frac{C_{s}}{\mu}-\bar{R} P_{s}\left(q_{k}\right)\right),
$$

where $P_{s}\left(q_{k}\right)$ is the probability that the tagged relay be the first one to transmit message $k$ to the destination, given the acceptance probability $q_{k}$ of the others. In (1), $C_{r}$ is the cost of accepting the message from the source and $C_{s} / \mu$ is the cost of storing the message until the relay meets the destination (recall that the inter-meeting times with the destination are exponentially distributed with mean $1 / \mu)$. The term $\bar{R} P_{s}\left(q_{k}\right)$ is the expected reward the relay gets the message. Thus, (1) gives the net expected cost for accepting the message.

For the tagged relay, the optimal value of $q_{k}^{\prime}$ is the one which minimizes (1). It follows that $q_{k}^{\prime}=0$ if $C_{r}+\frac{C_{s}}{\mu}-\bar{R} P_{s}\left(q_{k}\right)>0$. Hence, we conclude that if $\bar{R} \leq \bar{R}_{\text {min }}=C_{r}+\frac{C_{s}}{\mu}$, no relay will accept the $k^{t h}$ message. In other words, the condition $\bar{R}>\bar{R}_{\text {min }}$ is a necessary condition for the relays to have an incentive to participate in message delivery. Assuming that this condition is met, we see that $q_{k}^{\prime}=1$ is the best response of the tagged relay if $\bar{R}_{\min } / P_{s}\left(q_{k}\right)<\bar{R}$, while $q_{k}^{\prime}=q_{k}$ is one of the possible best responses if $\bar{R}_{m i n} / P_{s}\left(q_{k}\right)=\bar{R}$. We thus need to analyse how $P_{s}\left(q_{k}\right)$ depends on $q_{k}$.

To this end, let $p_{k}$ be the probability, as computed by the tagged relay, that an arbitrary other relay meets the source while it is proposing the $k^{t h}$ message and that this relay is not already busy with a previous message. Obviously, for the first message we have $p_{1}=1-e^{-\lambda T}$. The derivation of $p_{k}$ for $k>1$ is slightly more complex and we shall shortly explain how it can be computed by the tagged relay. From the definition of $p_{k}$, we obtain that $p_{k} q_{k}$ is the probability that an arbitrary other relay attempts the delivery of the $k^{\text {th }}$ message. Therefore, the number $A_{k}$ of other relays that are in competition with the tagged relay for the delivery of the $k^{t h}$ message follows a binomial distribution with parameter $p_{k} q_{k}$, 
which yields

$$
P_{s}\left(q_{k}\right)=\mathbb{E}\left(\frac{1}{A_{k}+1}\right)=\frac{1-\left(1-p_{k} q_{k}\right)^{N}}{N p_{k} q_{k}} .
$$

From (2), we can conclude that, if $\bar{R}>\bar{R}_{\text {min }}$, there exists a unique symmetric equilibrium with $q_{k}>0$, as formally stated in Theorem 1 below.

Theorem 1. If $\bar{R}>\bar{R}_{\text {min }}$, there exists a unique symmetric Nash equilibrium for the $k^{\text {th }}$ message with $q_{k}>0$. Moreover, we have $q_{k}=1$ if

$$
\bar{R}>\frac{N p_{k}}{1-\left(1-p_{k}\right)^{N}} \bar{R}_{m i n},
$$

while otherwise $q_{k}$ is the unique solution in $(0,1)$ of

$$
\bar{R}=\frac{N p_{k} q_{k}}{1-\left(1-p_{k} q_{k}\right)^{N}} \bar{R}_{\text {min }} .
$$

Proof. See Appendix A.

The structure of the Nash equilibrium is illustrated in Fig. 1 for the first message. If $\bar{R} \leq \bar{R}_{\text {min }}$, no relay accepts the message. If $\bar{R}>\frac{N\left(1-e^{-\lambda T}\right)}{1-e^{-\lambda N T}} \bar{R}_{\text {min }}$, at the unique Nash equilibrium all relays accept the message with probability 1. Otherwise, the relays use a randomized strategy with $0<q_{1}<1$.

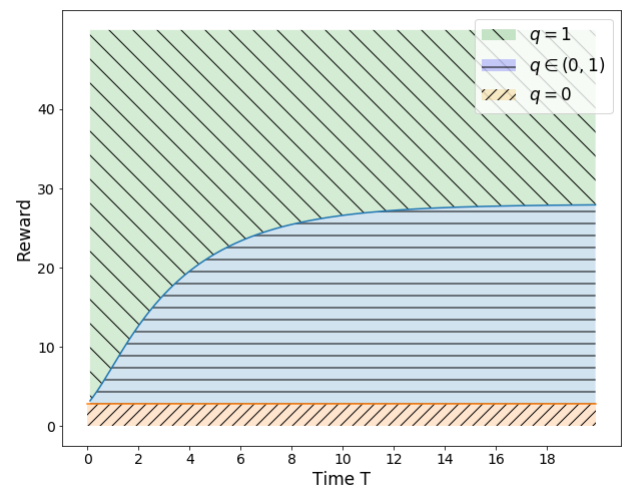

Fig. 1: Equilibrium acceptance probability $q_{1}$ as a function of $R$ and $T$, when the values of the parameters are as follows: $\mu=0.5, \lambda=0.3, C_{r}=C_{d}=2$ and $C_{s}=0.4$. 


\subsection{Computation of the probability $p_{k}$}

For the first message, we already know the value of $p_{1}$. We now explain how the value of $p_{k}$ can be computed by the tagged relay for subsequent messages $k>1$. We need to consider the belief of the tagged relay regarding the number of other relays that are in competition with it for the delivery of the $k^{t h}$ message. We assume that all relays play their equilibrium strategies $q_{i}, i=1, \ldots, k-1$ for all previous messages. Define $\Phi_{k}(t)$ as the probability that an arbitrary relay enters into competition for message $k$ on or before time $t$. By enter into competition on or before time $t$, we mean that there exists a time instant $t^{\prime}<t$ such that the considered relay does not have any message with index smaller than $k$ in the interval $\left[t^{\prime}, t\right]$. We shall denote by $\phi_{k}(t)$ the probability density function (pdf) corresponding to $\Phi_{k}(t)$. If this pdf is known by the tagged relay, then it can estimate the probability $p_{k}$ as follows.

$$
p_{k}=\int_{T(k-1)}^{T k} \phi_{k}(x)\left(1-e^{-\lambda(k T-x)}\right) d x .
$$

Denote by $\delta_{x}(t)$ the Dirac delta function at point $x$. Following the same approach as in [18], we can the following result.

Lemma 1. The density $\phi_{k}(t)$ obeys the recursion

$$
\phi_{k+1}(t)=h_{1}(k) \delta_{k T}(t)+\phi_{k}(t)+h_{2}(k) \mu e^{-\mu t} .
$$

Here $h_{1}(k)$ represents the probability that a relay is free for the $(k+1)^{t h}$ message at time $k T$, and is given by

$$
h_{1}(k)=\int_{(k-1) T}^{k T} \phi_{k}(x)\left(1-q_{k} I_{k}(x, k T)\right) d x,
$$

and $h_{2}(k) e^{-\mu k T}$ is the probability that a relay be busy with the $k^{t h}$ message at time $k T$, and is given by

$$
h_{2}(k)=e^{\mu k T} \int_{(k-1) T}^{k T} q_{k} \phi_{k}(x) I_{k}(x, k T) d x .
$$

In (6) and (7),

$$
I_{k}(x, t)=\frac{\lambda}{\mu-\lambda} e^{-\mu t} e^{\lambda x}\left(e^{(\mu-\lambda) \min (t, k T)}-e^{(\mu-\lambda) x}\right),
$$

represents the probability that a relay that comes into play at time $x<k T$ will meet the source and will not meet the destination by time $t$.

Since $h_{2}(i) e^{-\mu i T}$ is the probability that a relay has message $i$ at $i T$, it can be seen that $h_{2}(i) e^{-\mu(k-1) T}$ is the probability that a relay has message $i$ at time $(k-1) T$. Also, $h_{1}(k-1)$ is the probability that the relay does not have a message 
time $(k-1) T$. Since a relay either has a message or does not have one, we get the following relation:

$$
h_{1}(k-1)+e^{-\mu(k-1) T} \sum_{i=1}^{k-1} h_{2}(i)=1
$$

which yields

$$
\sum_{i=1}^{k-1} h_{2}(i)=\frac{1-h_{1}(k-1)}{e^{-\mu(k-1) T}} .
$$

Using (5)-(8) and induction, we can prove that $h_{1}(k)$ obeys the recursions given below. We omit the proof due to lack of space.

Proposition 1. The terms $h_{1}(k)$ can be computed with the recursion:

$$
\begin{aligned}
h_{1}(k)= & h_{1}(k-1)\left(1-q_{k} I_{k}((k-1) T, k T)\right)+\left(1-h_{1}(k-1)\right)\left(1-e^{-\mu T}\right) \\
& -\left(1-h_{1}(k-1)\right) \frac{q_{i} \lambda \mu e^{-\mu T}}{\mu-\lambda}\left(\frac{e^{(\mu-\lambda) T}-1}{\mu-\lambda}-T\right),
\end{aligned}
$$

with the initial value: $h_{1}(1)=1-q_{1} I(0, T)$. This leads to the following formulas for $h_{2}(k)$ and $p_{k}$ :

$$
\begin{aligned}
h_{2}(k) e^{-\mu k T}= & 1-h_{1}(k)-\left(1-h_{1}(k-1)\right) e^{-\mu T} \\
p_{k}= & h_{1}(k-1)\left(1-e^{-\lambda T}\right) \\
& +\left(1-h_{1}(k-1)\right)\left(1-e^{-\mu T}-\frac{\mu}{\mu-\lambda}\left(e^{-\lambda T}-e^{-\mu T}\right)\right) .
\end{aligned}
$$

Equation (9) has the following probabilistic interpretation. The probability that a relay can meet the source for message $k$ can be conditioned on two events at time $(k-1) T$ (i.e., at the release time of message $k$ ): either the relay did not have a message or had one of the previous $k-1$ messages. The two terms in (9) correspond to each of the two events. In the case of the first event, the probability of picking up message $k$ is just the probability of meeting the source in the interval $((k-1) T, K T]$. Since $h_{1}(k-1)$ is the probability of not having a message at time $(k-1) T$, the term $h_{1}(k-1)\left(1-e^{-\lambda T}\right)$ is the probability related to the first event. Next, we look at the second event. Suppose the relay has a message at time $(k-1) T$. It can take message $k$ only if it meets the destination and then the source in an interval of length $T$ starting from $(k-1) T$. This probability is the one inside the parenthesis of the second term in (9). Since $\left(1-h_{1}(k-1)\right)$, is the probability that the relay has a message at $(k-1) T$, the second term in (9) corresponds to the second event.

\subsection{Asymptotic Analysis when $k \rightarrow \infty$}

In this section, we shall do the analysis when $k$ is large, that is, when the system is in steady-state or stationary regime. In this regime, the function $\phi_{k}$ will 
reach its limiting value so that each message will have statistically the same performance measures. This regime reflects the long-run characteristics which are obtained after a large number of messages have been transmitted. From numerical experiments, it will be seen that, for our model, after as few as 10 to 15 messages, the system reaches the steady-state.

Let $h_{2}^{\prime}(k)=h_{2}(k) e^{-\mu k T}$. From Proposition 1 we get the following expressions for the limiting values of $p_{k}, h_{1}$, and $h_{2}^{\prime}$. The proof is omitted.

Proposition 2. When $k$ is large, we have

$$
\begin{aligned}
h_{1}(\infty):=h_{1} & =\frac{C(T)}{q_{\infty} I_{\infty}+C(T)}, \\
h_{2}^{\prime}(\infty):=h_{2}^{\prime} & =\left(1-h_{1}\right)\left(1-e^{-\mu T}\right), \\
p_{\infty} & =h_{1}\left(1-e^{-\lambda T}\right)+\left(1-h_{1}\right) D(T),
\end{aligned}
$$

where

$$
\begin{aligned}
C(T) & =1-e^{-\mu T}-\frac{q_{\infty} \mu \lambda}{\mu-\lambda}\left(\frac{e^{-\lambda T}-e^{-\mu T}}{\mu-\lambda}-T e^{-\mu T}\right), \\
D(T) & =1-e^{-\mu T}-\frac{\mu}{\mu-\lambda}\left(e^{-\lambda T}-e^{-\mu T}\right), \\
I_{\infty} & =\frac{\lambda}{\mu-\lambda}\left(e^{-\lambda T}-e^{-\mu T}\right) .
\end{aligned}
$$

From Proposition 2, we can write the relation between $q_{\infty}$ and $p_{\infty}$ as

$$
p_{\infty}\left(q_{\infty}\right)=\frac{C(T)\left(1-e^{-\lambda T)}\right.}{q_{\infty} I_{\infty}+C(T)}+\frac{q_{\infty} I_{\infty} D(T)}{q_{\infty} I_{\infty}+C(T)}
$$

Now, we can establish the conditions when $q_{\infty}=1$ and when $q_{\infty}<1$.

Lemma 2. If the following condition is satisfied, then $q_{\infty}=1, p_{\infty}=p_{\infty}(1)$ :

$$
\bar{R}-\frac{N p_{\infty}(1)\left(C_{r}+C_{s} / \mu\right)}{1-\left(1-p_{\infty}(1)\right)^{N}}>0
$$

Otherwise, $p_{\infty}$ and $q_{\infty}$ are the unique solution of the following system of equations:

$$
\begin{gathered}
\bar{R}-\frac{N p_{\infty} q_{\infty}\left(C_{r}+C_{s} / \mu\right)}{1-\left(1-p_{\infty} q_{\infty}\right)^{N}}=0 \\
\frac{C(T)\left(1-e^{-\lambda T)}\right.}{q_{\infty} I_{\infty}+C(T)}+\frac{q_{\infty} I_{\infty} D(T)}{q_{\infty} I_{\infty}+C(T)}=p_{\infty}
\end{gathered}
$$

The proof follows directly from Theorem 1 . Notice that in case of $q_{\infty}<1$, there is unique solution since the left hand side of (17) is decreasing in $q_{\infty}$.

Fig. 2 presents the probability $p_{k}$ that an individual relay, which is not busy with any previous message, meets the source while it is proposing the $k^{t h}$ message. This probability is computed from analytical expressions as well as from 
simulations for different values of $R, T=1.00357$ and $N=10$ (the other parameters have the same value as in Fig. 1). In fact, the value of $T$ is the value of $\hat{\theta}_{\infty}=\lim _{k \rightarrow \infty} \theta_{k+1}-\theta_{k}$ and the value of $R$ is expressed as a multiple of $R_{\text {min }}=\bar{R}_{\text {min }}+C_{d}=C_{r}+\frac{C_{s}}{\mu}+C_{d}$. The simulations consist of generating meeting times of relays with the source and the destination, then each relay deciding whether to accept or not the message when it meets the source, and then determining which relay wins the reward. The value of $p_{k}$ was then averaged over 2, 000 sample paths. For the same parameter values, Fig. 3 presents the acceptance probabilities $q_{k}$ as well as their limiting value $q_{\infty}$. From these figures, it can be seen that the steady-state is reached quite quickly (after 10 messages).

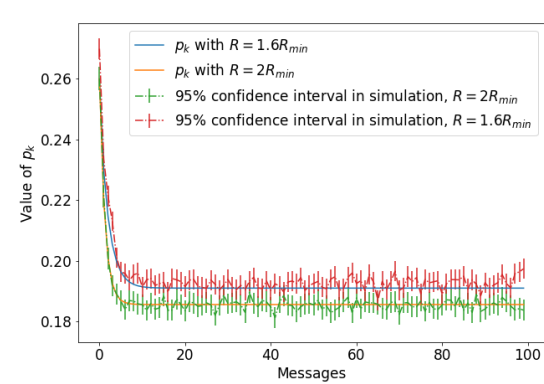

Fig. 2: Value of $p_{k}$.

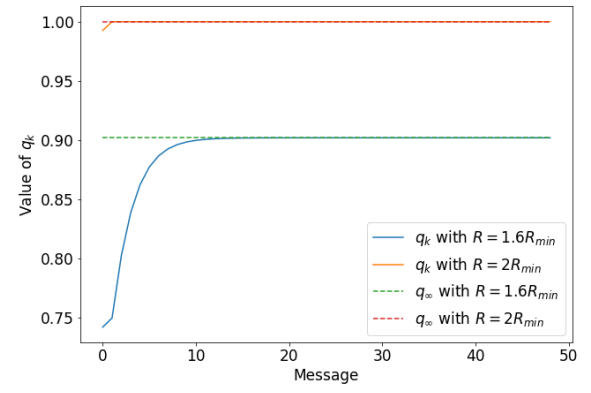

Fig. 3: Value of $q_{k}$ and its limiting value.

\section{Performance Metrics}

In this section, we use the results obtained in Section 3 to establish explicit expressions for the probability of message delivery and the mean time to deliver a message at the symmetric Nash equilibrium. Together with Theorem 1 and (9), our first result, formally stated in Proposition 3, allows to compute the probability of message delivery of each message.

Proposition 3. The probability of successful delivery of the $k^{\text {th }}$ message is $\xi_{k}=$ $1-\left(1-q_{k} p_{k}\right)^{N}$.

Proof. Each individual relay participates to the delivery of the $k^{t h}$ message with probability $q_{k} p_{k}$, from which the result follows.

Fig. 4 shows the probability of message delivery for different values of $R$, and the following parameter values: $T=1.00357$ and $N=10$. The other parameters are the same as in Fig. 1. The probabilities obtained with event-driven simulations are also shown in Fig. 4. In the simulation, we generate the inter-contact 
times between the source, the destination and relays. We then let the relays follow the mixed strategy with $q_{k}$ computed from previous sections. We run the simulation 5000 times and take the average.

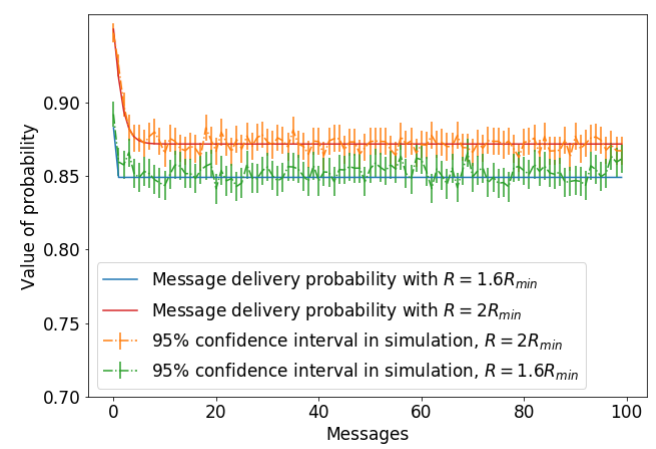

Fig. 4: Analytical probability of message delivery and simulated probability for different values of $\bar{R}$.

Proposition 4. Let $D_{k}$ denote the delay of the $k^{\text {th }}$ message. It holds that

$$
\mathbb{E}\left(D_{k} \mid D_{k}<\infty\right)=\frac{1}{\xi_{k}} \int_{(k-1) T}^{\infty}(1-Q(t))^{N}-\left(1-q_{k} p_{k}\right)^{N} d t
$$

where, with the notation $m=\min (t, k T), Q(t)$ is defined as

$$
Q(t)=q_{k} \int_{(k-1) T}^{m} \phi_{k}(x)\left[1-e^{-\lambda(m-x)}-I_{k}(x, t)\right] d x,
$$

and represents the probability that an individual relay will deliver the $k^{\text {th }}$ message by time $t$.

Proof. The probability that an individual relay that comes into play at time $x$ will meet the source by time $m \geq x$ and the destination by time $t \geq m$ is

$$
\int_{x}^{m} \lambda e^{-\lambda(s-x)}\left(1-e^{-\mu(t-s)}\right) d s=1-e^{-\lambda(m-x)}-I_{k}(x, t) .
$$

With $m=\min (t, k T)$, it follows that the probability that an individual relay will deliver the $k^{\text {th }}$ message by time $t$ is

$$
Q(t)=q_{k} \int_{(k-1) T}^{m} \phi_{k}(x)\left[1-e^{-\lambda(m-x)}-I_{k}(x, t)\right] d x,
$$


and hence the probability that the message is not delivered by time $t$ is $\mathbb{P}\left(D_{k}>t\right)=$ $(1-Q(t))^{N}$. The proof now follows from

$$
\begin{aligned}
\mathbb{E}\left(D_{k} \mid D_{k}<\infty\right) & =\int_{0}^{\infty} \mathbb{P}\left(D_{k}>t \mid D_{k}<\infty\right) d t \\
& =\frac{1}{\xi_{k}} \int_{0}^{\infty} \mathbb{P}\left(D_{k}<\infty\right)-\mathbb{P}\left(D_{k} \leq t\right) d t \\
& =\frac{1}{\xi_{k}} \int_{0}^{\infty} \mathbb{P}\left(D_{k}>t\right)-\left(1-q_{k} p_{k}\right)^{N} d t
\end{aligned}
$$

Fig. 5 shows the mean message delivery time for different values of $R$. The delays obtained with event-driven simulations are also shown on the figure. The parameter values are identical to those used in Fig. 4.

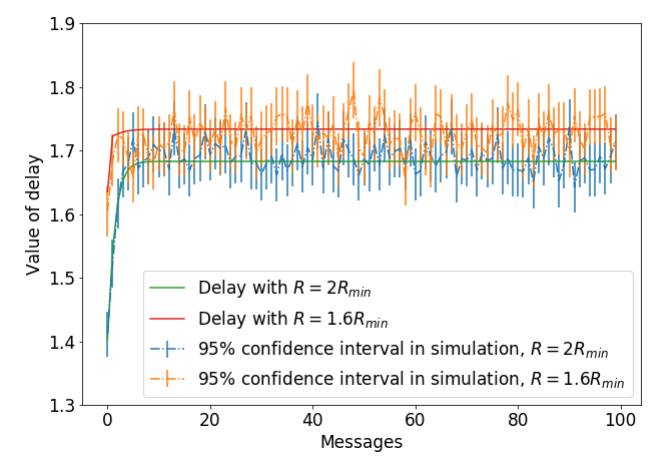

Fig. 5: Analytical delay and simulated delay.

\section{Comparison between the Threshold-type Strategy and the Randomized Policy}

In this section, we compare the performance obtained with the threshold-type strategy in the full information setting and with the randomized policy in the no information setting. We first consider the case where the source proposes each message for the same amount of time in both settings, that is, $T=\theta_{k}$ for the $k^{t h}$ message $\left(\theta_{k}\right.$ and $\gamma_{k}$ are the first and second thresholds, respectively, for the $k^{t h}$ message). Fig. 6 shows the structure of the Nash equilibrium strategies for the first message in both settings. It turns out that the randomized policy is either to reject the message $(q=0)$ or to accept it $(q=1)$ depending on the value of $R$, but independently of the value of $\lambda$. In contrast, the value of $\gamma$ in the 
threshold-type policy depends on the value of $\lambda$. We emphasize that when $q=1$ and $\gamma=\infty$, the two policies coincide: all relays accept the message as long as it is proposed by the source and keep it until they meet the destination (this is not the case when $\gamma<\infty$ since relays can drop the message before meeting the destination). Therefore, in this situation, the source does not need to provide the birth-time of its messages. Moreover, the relays do not need to take care of time, they just decide to accept a message or not, and then keep the message until meeting the destination. Fig. 7 compares the message delivery probabilities

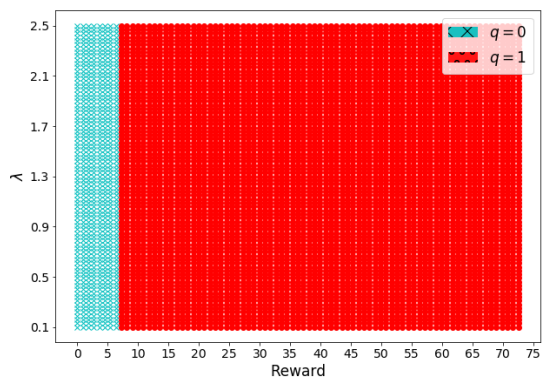

(a) Randomized Policy

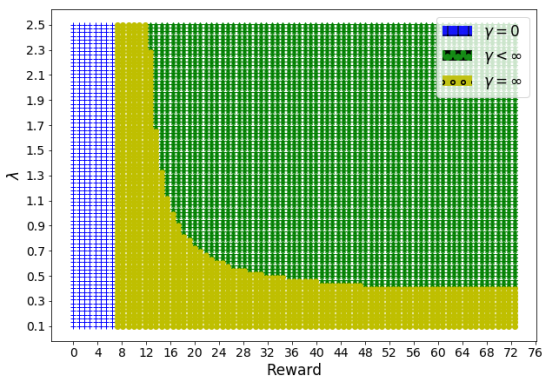

(b) Threshold-type Policy

Fig. 6: Randomized and threshold-type policies as functions of $R$ and $\lambda$ for the first message when $T=\theta_{1}$. The values of the parameters are $\mu=0.4, C_{d}=2$, $C_{r}=4, C_{s}=0.5$ and $N=3$.

in both settings as $T$ varies. In this case, we consider the steady-state message delivery probabilities, which are obtained as $k \rightarrow \infty$, for two different values of $R$. The figure also shows the asymptotic value of the acceptance probability $q_{\infty}$ in the no information setting. For $R=2 R_{\text {min }}=10$, we have $\theta=0.65$ and $\gamma=\infty$ for the threshold policy. We observe that the message delivery probability in the no information setting increases as $T$ grows: for $T \leq \theta$, the acceptance probability $q_{\infty}=1$ and the message delivery probability is lower than in the full information setting. Both settings coincide when $T=\theta$, as expected. For $T>\theta$, the acceptance probability $q_{\infty}<1$, but the message delivery probability keeps increasing until it reaches its limiting value, which is higher than in the full information setting. For $R=3.5 R_{\min }=17.5$, we have $\theta=0.91$ and $\gamma=3.07$. We observe a similar behavior of the message delivery probability in the no information setting, despite the fact that in this case $\gamma<\infty$. These results suggest that by using a value of $T$ slightly larger than $\theta$, and for the same reward value $R$, the source can increase its message delivery probability if it does not tell the relays when a message was generated. 


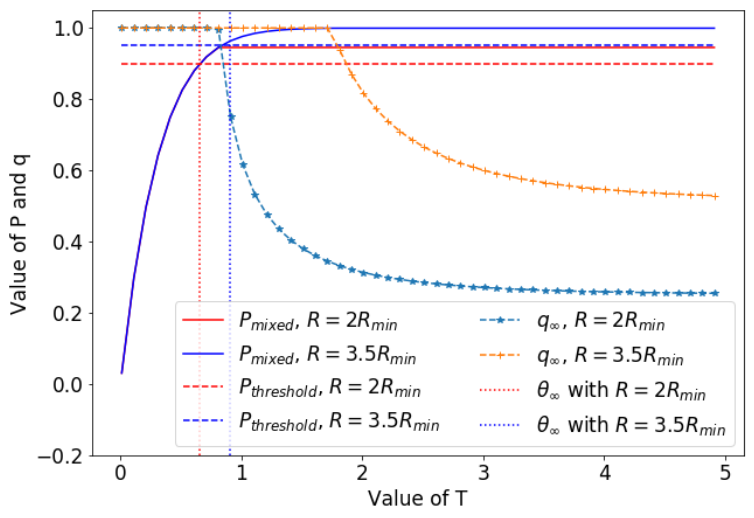

Fig. 7: The message delivery probability in mixed strategy and threshold strategy, with $\mu=0.4, C_{d}=C_{r}=2, C_{s}=0.4, N=10$ and $\lambda=1.5$.

\section{Conclusions}

We analyzed a competitive DTN game between $N$ relays in which the source does not give information on the message generation times to the relays. The equilibrium obtained is a mixed one in which a relay accepts a message with a certain probability. This contrasts with the threshold-based equilibrium in [18] in which the source gave message generation information to the relays. Simulations suggest giving no information on the message generation times can be advantageous to the source compared to giving information. By taking the duration for which a message is proposed to be slightly longer than the equilibrium threshold in [18], the source can improve the limiting value of its message delivery probability.

\section{Acknowledgements}

We thank the anonymous referees for their constructive comments that have helpful in improving the quality of the paper.

\section{References}

1. Altman, E.: Competition and Cooperation between Nodes in Delay Tolerant Networks with Two Hop Routing, pp. 264-278. Springer Berlin Heidelberg, Berlin, Heidelberg (2009)

2. Basilico, N., Cesana, M., Gatti, N.: Algorithms to find two-hop routing policies in multiclass delay tolerant networks. IEEE Transactions on Wireless Communications 15(6), 4017-4031 (June 2016). https://doi.org/10.1109/TWC.2016.2532859 
3. Benhamida, F.Z., Bouabdellah, A., Challal, Y.: Using delay tolerant network for the internet of things: Opportunities and challenges. In: 2017 8th International Conference on Information and Communication Systems (ICICS). pp. 252-257 (April 2017). https://doi.org/10.1109/IACS.2017.7921980

4. Buttyan, L., Dora, L., Felegyhazi, M., Vajda, I.: Barter-based cooperation in delaytolerant personal wireless networks. In: IEEE International Symposium on a World of Wireless, Mobile and Multimedia Networks (2007)

5. Buttyan, L., Dora, L., Felegyhazi, M., Vajda, I.: Barter trade improves message delivery in opportunistic networks. Ad Hoc Networks 8(1), 1-14 (January 2010)

6. Cai, H., Eun, D.: Crossing over the bounded domain: From exponential to powerlaw inter-meeting time in MANET. In: Proceedings of ACM/IEEE MOBICOM (2007)

7. Chahin, W., Sidi, H.B., El-Azouzi, R., Pellegrini, F.D., Walrand, J.: Incentive mechanisms based on minority games in heterogeneous DTNs. In: Proceedings of 25th ITC conference. Sanghai, China (Sept 10-12 2013)

8. Chen, B.B., Chan, M.C.: Mobicent: a credit-based incentive system for disruption tolerant network. In: 2010 Proceedings IEEE INFOCOM. pp. 1-9 (March 2010). https://doi.org/10.1109/INFCOM.2010.5462136

9. Fall, K.: A delay-tolerant network architecture for challenged internets. In: Proc. ACM SIGCOMM, Karlsruhe, Germany. pp. 27-34 (2003). https://doi.org/10.1145/863955.863960

10. Giannini, C., Calegari, P., Buratti, C., Verdone, R.: Delay tolerant network for smart city: Exploiting bus mobility. In: 2016 AEIT International Annual Conference (AEIT). pp. 1-6 (Oct 2016). https://doi.org/10.23919/AEIT.2016.7892779

11. Giannini, C., Shaaban, A.A., Buratti, C., Verdone, R.: Delay tolerant networking for smart city through drones. In: 2016 International Symposium on Wireless Communication Systems (ISWCS). pp. 603-607 (Sept 2016). https://doi.org/10.1109/ISWCS.2016.7600975

12. He, Q., Wu, D., Khosla, P.: SORI: A secure and objective reputation-based incentive scheme for ad hoc networks. In: Proceedings of IEEE WCNC (2004)

13. Ito, M., Nishiyama, H., Kato, N.: A novel routing method for improving message delivery delay in hybrid DTN-MANET networks. In: 2013 IEEE Global Communications Conference (GLOBECOM). pp. 72-77 (Dec 2013). https://doi.org/10.1109/GLOCOM.2013.6831050

14. Mahmoud, M.E., Shen, X.: PIS: A practical incentive system for multi-hop wireless networks. IEEE Transactions on Vehicular Technology pp. 4012-4025 (2010)

15. Malathi, M., Jayashri, S.: Design and performance of dynamic trust management for secure routing protocol. In: 2016 IEEE International Conference on Advances in Computer Applications (ICACA). pp. 121-124 (Oct 2016). https://doi.org/10.1109/ICACA.2016.7887935

16. Marti, S., Giuli, T.J., Lai, K., Baker, M.: Mitigating routing misbehavior in mobile ad hoc networks. In Proc. of MobiCom pp. 255-265 (2000)

17. Nguyen, T.T.H., Brun, O., Prabhu, B.J.: Mean-field limit of the fixed-reward incentive mechanism in delay tolerant networks. In: 2018 16th International Symposium on Modeling and Optimization in Mobile, Ad Hoc, and Wireless Networks (WiOpt). pp. 1-8 (May 2018). https://doi.org/10.23919/WIOPT.2018.8362810

18. Nguyen, T.T.H., Brun, O., Prabhu, B.: Performance of a fixed reward incentive scheme for two-hop DTNs with competing relays (long version) (Aug 2017), https://hal.laas.fr/hal-01575320, this is the long version of the NetEcon paper http://netecon.eurecom.fr/NetEcon2016/papers/Nguyen.pdf which is also in HAL https://hal.archives-ouvertes.fr/hal-01365939 
19. Seregina, T., Brun, O., Elazouzi, R., Prabhu, B.: On the design of a reward-based incentive mechanism for delay tolerant networks. IEEE Transactions on Mobile Computing 16(2), 453-465 (2017)

20. Shevade, U., Song, H., Qiu, L., Zhang, Y.: Incentive-aware routing in DTNs. In: IEEE International Conference on Network Protocols (ICNP). pp. 238-247 (2008)

21. Torabkhani, N., Fekri, F.: Delay analysis of bursty traffic in finite-buffer disruptiontolerant networks with two-hop routing. In: 2013 IEEE International Conference on Sensing, Communications and Networking (SECON). pp. 541-549 (June 2013). https://doi.org/10.1109/SAHCN.2013.6645026

22. Uddinand, M.Y.S., Godfrey, B., Abdelzaher, T.: RELICS: In-network realization of incentives to combat selfishness in DTNs. In: Proceedings of IEEE International Conference on Network Protocols (ICNP). pp. 203-212 (2010)

23. Wei, L., Cao, Z., Zhu, H.: MobiGame: A user-centric reputation based incentive protocol for delay/disruption tolerant networks. In: Proc. IEEE Global Telecommunications Conference (GLOBECOM). pp. 1-5 (2011)

24. Zhang, X., X.Wang, Liu, A., Zhang, Q., Tang, C.: Reputation-based schemes for delay tolerant networks. In: Proceedings of 2011 International Conference on Computer Science and Network Technology (2011)

25. Zhong, S., Chen, J., Yang, Y.R.: Sprite, a simple, cheat-proof, credit-based system for mobile ad-hoc networks. In: Proceedings of INFOCOM 2003. pp. 1987-1997. San Francisco, CA, USA (April 2003)

26. Zhu, H., Lin, X., Lu, R., Fan, Y., Shen, X.: SMART: A secure multilayer creditbased incentive scheme for delay-tolerant networks. IEEE Transactions on Vehicular Technology 58(8), 4628-4639 (2009)

\section{A Proof of Theorem 1}

Before proving the lemma, we first prove that the probability $P_{s}\left(q_{k}\right)$ is decreasing in $q_{k}$. With $r=p_{k} q_{k}$, we have

$$
\frac{\partial P_{s}\left(q_{k}\right)}{\partial q_{k}}=\frac{N r(1-r)^{N-1}-1+(1-r)^{N}}{(N r)^{2}}
$$

The numerator is negative since it has value 0 when $r=0$ and it is decreasing in $r$ (the derivative w.r.t $r$ is negative), and thus in $q_{k}$. It follows that the expected net cost $\bar{R}_{\text {min }}-\bar{R} P_{s}\left(q_{k}\right)$ is increasing in $q_{k}$ and reaches its maximum value for $q_{k}=1$.

Assume $\bar{R}>\bar{R}_{\text {min }}$. If the other relays play $q_{k}=1$, the best-response strategy of the tagged relay is $q_{k}^{\prime}=1$ if and only if $\bar{R}_{m i n}-\bar{R} P_{s}(1)<0$, which is equivalent to $(3)$. On the other hand, for $q_{k} \in(0,1)$ to be a symmetric equilibrium, $\bar{R}_{\text {min }}-$ $\bar{R} P_{s}\left(q_{k}\right)=0$ must hold, which is equivalent to (4). It is easy to see from (4) that $\bar{R}$ is an increasing function of $q_{k}$ such that $\bar{R} \in\left[\bar{R}_{\min }, \bar{R}_{\max }\right]$, where $\bar{R}_{\max }=$ $\frac{N q_{k} p_{k}}{1-\left(1-q_{k} p_{k}\right)^{N}} \bar{R}_{\min }$. Therefore, there is a bijective function between $\bar{R}$ and $q_{k}$. Hence, for any $\bar{R} \in\left[\bar{R}_{\min }, \bar{R}_{\max }\right]$, we always can find a value of $q_{k}$ such that the equation (4) is satisfied. 